\title{
My memories of E. C. G. (George) Sudarshan
}

\author{
Sheldon Lee Glashow*
}

George Sudarshan came to the Physics Department at Harvard, USA as its Corporation Fellow in the fall of 1957 while I was a graduate student under Julian Schwinger. Both of us, George and I, focused on the theory of weak interactions and soon became fast friends. We were both puzzled by the relative weakness of strangeness-changing beta decays, coming close - but not close enough - to the notion of an angle to preserve the universality of weak forces, first proposed by Gell-Mann and Lévy in 1960 in the context of the Sakata model, and later extended to the unitary symmetry scheme by Cabibbo.

I cannot refrain from mentioning one amusing incident from that time. Generally bachelor physicists like us would inhabit small off-campus furnished apartments. George, however, resided at the local YMCA. He explained this odd choice by noting that as a rather privileged youth he was never called upon to tie his own shoes. Fortunately, the YMCA was able to provide this essential service! Whether this tale be true or not I cannot say: George's sense of humour was commensurate with his scientific imagination.

The mid-1950s were a time of great ferment and confusion in particle physics. The newly discovered failure of mirror symmetry reinvigorated the study of nuclear beta decay, while the decay properties of pions and strange particles were just being studied. How were the new results to be incorporated into Fermi's decades-old framework? To make matters worse, no one model seemed capable of agreeing with all of the data. T. D. Lee concluded that 'a universal Fermi interaction could not be realized', while Schwinger, choosing the wrong data to ignore, published a deeply flawed version of a universal theory of weak interactions. Marshak and Sudarshan, having pondered over these matters for some months, met with Felix Boehm in July 1957, where they learned that the latest data did not favour the VT possibility that Schwinger had chosen. They soon were able to complete and submit their ground-breaking paper 'The nature of the four-fermion interaction', of which Sudarshan, then Marshak's graduate student, was the first-named author.

Sudarshan and Marshak presented a comprehensive analysis of weak interaction data, which along with the imposition of an elegant symmetry principle, allowed them to deduce a unique form for the weak interactions, the so-called V-A theory. Their daring hypothesis was accompanied by a list of four experimental results, that

*e-mail: slg@bu.edu they wrote, 'cannot be reconciled with this hypothesis ... All of these experiments should be redone ... If any of the four experiments stand, it will be necessary to abandon the hypothesis'. This is theoretical physics at its zenith! The experiments were redone with results that now confirm the hypothesis.

The V - A theory proposed by Sudarshan and Marshak became the textbook description of weak interactions, and it was the basis for the subsequent development of today's electroweak theory. It was a stunning accomplishment, yet one which has never been recognized with a prize.

The Sudarshan-Marshak (SM) paper was submitted practically at the same time as the Feynman-Gell-Mann (FG) paper, viz. 'Theory of the Fermi Interaction'. The two papers cover much the same ground. They offer somewhat different arguments leading to the same V-A theory, and both identify the same conflicting experiments. However, several reasons underlie my belief that Sudarshan and Marshak deserve priority in this matter:

(1) The FG paper includes the footnote: 'A universal $\mathrm{V}$ - A interaction has also been proposed by Sudarshan and Marshak (to be published).' This surely suggests that FG had access to the work of SM. In contrast, the SM paper, submitted on the very same day, includes merely the polite acknowledgement, 'We are grateful to Prof. Gell-Mann ... for valuable discussions'. These facts are consonant with Sudarshan's later claim 'that Gell-Mann was informed of our work on the $\mathrm{V}-\mathrm{A}$ theory [by] the first week of July, 1957, at which time our (SM) paper was completed.'

(2) Richard Feynman, himself a protagonist in the priority issue, made the following public and published remark much later, in 1974: 'We have a conventional theory of weak interactions [the $\mathrm{V}-\mathrm{A}$ theory] invented by Marshak and Sudarshan [sic], published by Feynman and Gell-Mann and completed by Cabibbo.'

Sadly, priority issues plagued Sudarshan several times during his professional career. In the early 1960s, he turned his attention to the quantum nature of coherent light. His work, shortly followed by that of Roy Glauber, led to the so-called Glauber-Sudarshan representation to describe light in optical phase space. This work was the subject of another priority controversy when Glauber was awarded half of the 2005 Nobel Prize in Physics for his work in this field and Sudarshan's contribution was not recognized. A petition to the Royal Swedish Academy submitted by a group of physicists read that it is difficult 
to understand how the work for which Glauber is cited could be honoured in isolation from Sudarshan's published discoveries ... which were initially criticized and subsequently adopted by Glauber'. Twice in his career, and in both cases for sensible reasons, Sudarshan felt himself unjustly deprived of a well-deserved Nobel Prize.

Sudarshan's significant contributions to physics span many other subdisciplines, from quantum measurement theory, formal field theory, primordial nucleosynthesis, faster-than-light particles, quantum computers and more. I am much less familiar with these issues because I left Harvard for Copenhagen in the summer of 1958, while Sudarshan accepted a position at Rochester, USA in 1959. Our paths crossed only a few times in the succeeding decades, but always with time to share happy memories.

doi: $10.18520 / \mathrm{cs} / \mathrm{v} 116 / \mathrm{i} 2 / 199-200$ 